\title{
Alopecia Universalis: an extremely rare but probable adverse effect of adalimumab
}

\author{
Pooja Kadam, MBBS ${ }^{(1)}$ Prashant Kaushik, MD ${ }^{(2)}$ \\ (1) Senior Resident Medical Officer, St Vincent's Hospital, 390 Victoria St, Darlinghurst NSW 2010, \\ Sydney, Australia \\ ${ }^{(2)}$ Department of Internal Medicine, Division of Rheumatology, Samuel S. Stratton Veterans Affairs Medical \\ Center, 113 Holland Ave, Albany, NY 12208 \\ Associate Professor, Department of Internal Medicine, Division of Rheumatology \\ Albany Medical College, Albany, NY
}

\begin{abstract}
Objective: To report alopecia universalis (AU) as an adverse effect of adalimumab that has not yet been documented in literature.

Case Summary: A 50-year-old male was diagnosed with psoriasis and psoriatic arthritis and was commenced on adalimumab subcutaneous injections biweekly. The psoriatic skin lesions and arthritis showed improvement. However, one year later he lost all his body hair including pubic hair. Trichoscopy confirmed AU. Adalimumab was believed to have caused this adverse drug reaction (ADR). He was switched to etanercept with a good response (psoriasis and psoriatic arthritis). However he had no evidence of hair regrowth six months after change of therapy.

Discussion: The exact pathogenesis of adalimumab causing hair loss is poorly understood. The patient's history of psoriasis and psoriatic arthritis conferred an autoimmune diathesis. Adalimumab with its immunomodulatory properties might have been the potential trigger for the development of AU with a sustained or even a permanent injury to the hair follicle as evidenced by absence of hair regrowth at 6-month follow up. Our patient scored a 7 on the Naranjo scale, which signifies that an ADR is the probable cause of AU.

Conclusion: This case highlights that AU is a probable ADR of adalimumab. Further studies are needed to assess the duration and reversibility of hair loss. Patients should be promptly educated regarding its occurrence prior to commencement of therapy in order to minimize any associated psychological impact.
\end{abstract}

Keywords: Alopecia universalis, Adalimumab, TNF-inhibitors (TNF-i), adverse drug reaction (ADR)

\section{INTRODUCTION}

$\mathrm{AU}$, an extreme form of AA is an autoimmune disease thought to be a Th1-lymphocyte mediated inflammation directed against an unknown autoantigen of the hair follicle. The exact underlying mechanism is thought to be a genetic predisposition relating to HLA antigen presenting molecules, changes in neuropeptide expression and an amendment in the immune-privileged state of the hair follicle ${ }^{1}$. In recent times TNF-i (TNFinhibitors) have demonstrated both efficacy and tolerability in patients with moderate-to-severe plaque psoriasis and associated arthritis ${ }^{2}$. We present a case of a patient, who developed AU during treatment of psoriasis with adalimumab which is a monoclonal antibody and binds specifically to the TNF-alpha receptor.

\section{CASE REPORT}

A 50-year-old male presented to his primary care physician with scaly, raised lesions on his elbows, knees and distal interphalangeal (DIP) joints. He also reported bilateral knee and elbow pain. The plaques on his extensor surfaces appeared more than 20 years ago, however the joint pain that started approximately 2 months ago prompted medical attention. He was referred to a dermatologist for further care and management by his family physician. He denied any injuries, chills, headaches or rashes elsewhere. He had a history of throat cancer that was treated using surgery and radiation. Subsequently he developed hypothyroidism secondary to radiation therapy and was commenced on levothyroxine. Other medications included omeprazole and occasional multivitamins. He was a previous smoker with a 50-pack year smoking history. He reported social alcohol consumption but denied recreational drug use. He worked as clerk previously and was now retired.

On examination, his vital signs were normal. Physical examination revealed scaly, erythematous plaques and papules on his elbows, knees and IPJ. Nail changes including pitting, subungual hyperkeratosis, discoloration and onycholysis were noted on several finger and toenails (Fig 1). There was tenderness to palpation at the 
elbows and DIP joints. The remainder of the physical examination was unremarkable. Complete metabolic panel and complete blood count were within normal limits.

He was diagnosed with psoriasis and psoriatic arthritis and started on adalimumab 40mg subcutaneously biweekly. Methotrexate was considered as the initial disease modifying anti-rheumatic drug, however, due to his continued alcohol use it was deferred. He responded to the therapy well (both psoriasis and inflammatory arthritis) and had no ADRs in the first year of treatment. However in the next few months he started noticing hair loss from his scalp, extremities and eyebrows and eventually lost all his hair within a month (Fig 2). AU was diagnosed and confirmed with trichoscopy. After a careful exclusion of other autoimmune causes of AU, it was suspected to be an ADR to adalimumab. Adalimumab was therefore replaced by etanercept, another TNF-i with a different structure though. Psoriasis and psoriatic arthritis continue to respond to etanercept without any other ADR. However over the next six months he had no evidence of any hair regrowth. Suspension of treatment with TNF alpha antagonists was considered. However, given the excellent response to adalimumab initially and since the patient did not wish to be treated with minoxidil or any other topical hair restoring agents, etanercept as a TNF-i with a different structure (a receptor fusion protein rather than a monoclonal antibody) was initiated.

Figure 1: Bilateral nail changes including pitting, subungual hyperkeratosis, discoloration and onycholysis are noted on his fingers

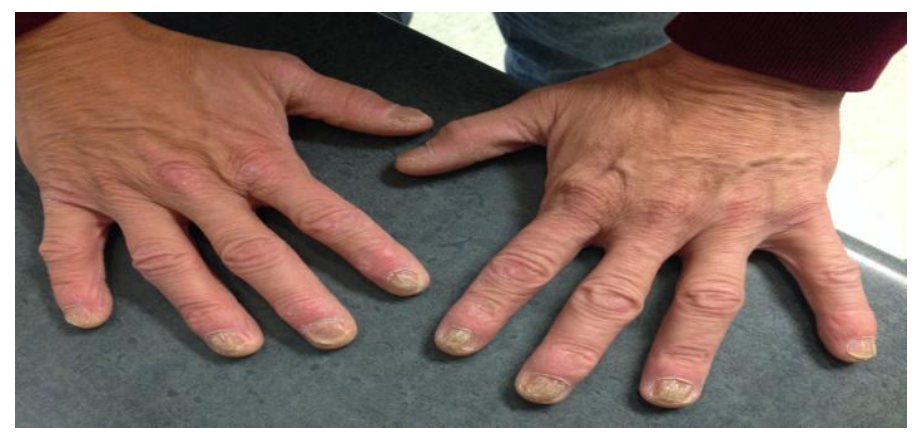

Figure 2: Alopecia universalis with complete hair loss of scalp and eyebrows.



III. DISCUSSION

Biological response modifiers such as TNF-i are increasingly being used for psoriatic arthritis when patients fail to respond adequately to or are intolerant of traditional disease-modifying anti-rheumatic drugs (DMARDs). Common ADRs of these agents include serious infections including reactivation of infections such as tuberculosis and fungal infections, heart failure, central demyelinating syndromes etc. These ADRs can be minimized with meticulous screening before initiation, regular clinical monitoring and patient education. The development of AA during treatment with TNF-i such as adalimumab for psoriasis has been previously reported $^{3}$. Our case extends the very recently reported association of adalimumab in rheumatoid arthritis with AU to the occurrence of the same ADR with the use of adalimumab in psoriatic arthritis, another autoimmune rheumatic disease ${ }^{4}$. More so, AU can be considered a class-effect of TNF-i because it did not reverse with the use of etanercept that belongs to the same class (TNF-i) with a different molecular structure.

This patient's course during biological response modifier therapy could be ascribed to a hypersensitivity immune-mediated ADR triggered by the drug. There is convincing evidence to suggest that patients with psoriasis and psoriatic arthritis have a greater risk of developing other autoimmune disorders, especially 
alopecia. Both diseases are autoimmune pathogenetically, and both show response to conventional immunosuppressive therapy, psoriasis more than AA. Our patient achieved significant improvement in psoriasis and psoriatic arthritis upon treatment with adalimumab. However, after a year of treatment he developed AA confirmed by trichoscopy. Within the next month his AA rapidly progressed to AU. What draws attention to our case is that the patient did not have any evidence of AU despite having psoriasis for over 30 years. More so, within a year of introducing adalimumab he developed AA, which rather quickly progressed to AU. This temporal association favors a cause and effect relationship between adalimumab and AU although we cannot exclude a coincidental occurrence of these two disorders. According to the Naranjo adverse drug reaction scalethat assesses the probability that an adverse event is related to drug therapy based on a list of weighted questions, our patient scored a 7 [Are there previous conclusive reports on this reaction? Yes $(+1)$; Did the adverse events appear after the suspected drug was given? Yes (+2); Are there alternative causes that could have caused the reaction? No $(+2)$; Did the patient have a similar reaction to the same or similar drugs in any previous exposure? Yes $(+1)$; Was the adverse event confirmed by any objective evidence? Yes $(+1)$. This reflects that AU was a probable ADR to the use of adalimumab $]^{5}$.

The Renbök phenomenon or inverse Köebner phenomenon refers to normal hair growth in psoriatic plaques observed in patients with psoriasis. Development of AU with TNF-i therapy such as adalimumab for psoriasis shows features of a "biological drug-induced Renbök phenomenon". The pathophysiological mechanism of this phenomenon remains unknown. It has been postulated that successful therapy of psoriasis with TNF-i may contribute to an 'immune switch', which facilitates $\mathrm{AU}^{6}$.

The patient had no evidence of hair regrowth at 6 months despite ceasing adalimumab therapy. This could suggest permanent hair loss or extremely slow hair regrowth, which could be a 'class-effect' since the loss of persisted with the use of etanercept, another TNF-i.

\section{CONCLUSION}

In this case we report AA quickly followed by AU as a probable ADR to the use of adalimumab for psoriasis and psoriatic arthritis. Meticulous selection of biological treatment regimens is therefore required to offer therapeutic benefits for psoriasis. With the growing use of TNF-i, it is prudent to be cognizant of this ADR and educate patients appropriately.

\section{REFERENCES}

[1]. Hordinsky MK. Overview of Alopecia Areata. J InvestigDermatolSympProc2013;16, S13-S15.

[2]. Strober B. Biologic therapy for psoriasis: early response implies future success. The Br J Dermatol 2013; 169(6):1178-9.

[3]. Zschoche C, Bidier M, Hadaschik E. Alopecia areata during treatment with adalimumab: therapy with an alternative TNF-alpha inhibitor is possible.J DtschDermatolGes. 2013; 11(5):450-3

[4]. Lazzarini R, Capareli GC, Buense R, Lellis RF. Alopecia universalis during treatment with leflunomide and adalimumab - case report. An Bras Dermatol. 2014; 89 (2):320-2.

[5]. Naranjo CA, Busto U, Sellers EM et al. A method for estimating the probability of adverse drug reactions. Clin. Pharmacol Ther 1981;30 (2): 239-45.

[6]. Garnacho-Saucedo GM, Salido-Vallejo R, Alvarez-Lopez MA, Casas de la Asuncion E, Ruano-Ruiz J, Garcia-Nieto AV et al. Renbök phenomenon in a patient with alopecia areatauniversalis. Arch Dermatol2012;148(8):964-5. 